\title{
The Regularities of Electrolytic Dissociation of Weak Dibasic and Tribasic Organic Wine Acids
}

\author{
E. Kvaratskhelia*, R. Kvaratskhelia and R. Kurtanidze
}

\section{R. Agladze Institute of Inorganic Chemistry and Electrochemistry, Ivane Javakhishvili Tbilisi State University, Tbilisi, Georgia}

\begin{abstract}
The weak dibasic and tribasic organic acids in wine: tartaric, malic, citric and succinic acids are the important components having direct influence on various properties of wine. The regularities of electrolytic dissociation of these acids determine all their useful properties. With the aid of suggested by authors original method for analysis the complex equilibria of the processes of dissociation of weak multibasic organic acids the main dissociation parameters of tartaric, malic, citric and succinic acids in their dilute solutions: the values of usual and "partial" degrees of dissociation of all steps, the concentrations of all anions, hydrogen ions and undissociated acid molecules are determined. The concentration intervals of predominance of various charged and uncharged substances in dilute solutions of all above mentioned acids are also determined. The simple empirical equations for fast approximate calculation of the dissociation degrees and $\mathrm{pH}$ values are also suggested.
\end{abstract}

Keywords: Tartaric acid, malic acid, citric acid, succinic acid, degree of dissociation, hydrogen ions concentration, dissociation step, dissociation constants, monoanion, dianion.

\section{INTRODUCTION}

The major wine acids are the weak multibasic organic acids: dibasic tartaric, malic, succinic and tribasic citric acids. They are the important components in both winemaking and the finished product of wine. These acids present in grapes and wine, having direct influence on the color, balance and taste of the wine as well as the growth and vitality of yeasts during fermentation and protecting the wine from bacteria. Taking into account that the hydrogen ions determining the acidity and other important properties of wine originate from dissociation of above mentioned acids, the regularities of the processes of electrolytic dissociation of the wine acids are of vital importance. These processes are characterized by complex "overlapping" equilibria because of the close values of the dissociation constants of separate steps of dissociation of wine acids.

In recent years we suggested the original method for analysis of the processes of electrolytic dissociation of weak multibasic organic acids with the "overlapping" equilibria effect [1-4]. The equations obtained by us give the opportunity to direct calculate such important dissociation parameters as usual and "partial" degrees of dissociation for all steps (the term of "partial" dissociation degree was first suggested by us: [3]), the concentrations of all anions, hydrogen ions and undissociated acid molecules, the concentration

*Address correspondence to this author at the R. Agladze Institute of Inorganic Chemistry and Electrochemistry, Ivane Javakhishvili Tbilisi State University, 11 Mindely st, 0186 Tbilisi, Georgia; Tel: 995595447870 ;

E-mail: elicko@mail.ru intervals of predominance of various charged and uncharged substances. Our method was successfully used for description of the regularities of electrolytic dissociation of various weak multibasic organic acids $[2,4,6,7]$. In this communication our method is used for an analysis of the peculiarities of electrolytic dissociation of the multibasic organic wine acids in their dilute solutions.

\section{GENERAL EQUATIONS}

The general mass action equations suggested by us for $m$ dissociation step of weak multibasic organic acid $\mathrm{H}_{\mathrm{n}} \mathrm{A}$ with the close values of dissociation constants of separate steps is written as follows:

$K_{m}=\frac{c\left(\alpha_{m}-\alpha_{m+1}\right) \sum_{m=1}^{n} \alpha_{m}}{\alpha_{m-1}-\alpha_{m}} F_{m}=\frac{c \alpha_{m}^{\prime}\left(1-\alpha_{m+1}^{\prime}\right) \sum_{m=1}^{n} \alpha_{m}^{\prime} \alpha_{m-1}}{1-\alpha_{m}^{\prime}} F_{m}$

where $\mathrm{K}_{\mathrm{m}}$ is the thermodynamic dissociation constant of $m$ step, $\alpha_{m}, \alpha_{m+1}$ and $\alpha_{m-1}$ are the usual degrees of dissociation of corresponding steps (they are equal to the ratio of the number of moles of $\mathrm{H}^{+}$ions formed in a given dissociation step to the total number of moles of acid), $\alpha_{m}^{\prime}, \alpha_{m+1}^{\prime}$ and $\alpha_{m-1}^{\prime}$ are the corresponding "partial" degrees of dissociation (they are equal to the ratio of the number of moles of anion dissociated at a given step to the number of moles of this anion formed in the previous step; for first step the concepts of usual and "partial" degrees of dissociation coincide), $\mathrm{c}$ is a total concentration of acid, $F_{m}$ is the quotient of the activity coefficients for $m$ step: 


$$
F_{m}=\frac{f_{H^{+}} f_{H_{n-m} A^{m-}}}{f_{H_{n-(m-1)} A^{(m-1)-}}}
$$

The values of the activity coefficients can be approximated by the Debye-Huckel equation:

$\lg f_{i}=-\frac{z_{i}^{2} A \sqrt{I}}{1+a_{i} B \sqrt{I}}$

where $a_{i}$ is the cation-anion distance of closest approach, $\mathrm{A}$ and $\mathrm{B}$ are constants depending on the properties of water at given temperature, $z_{i}$ is the charge of ion. The ionic strength $I=c \sum_{m=1}^{n} m \alpha_{m}=c \sum_{m=1}^{n} m \alpha_{m}^{\prime} \alpha_{m-1}$. The values of $\mathrm{A}$ and $\mathrm{B}$ at $25^{\circ} \mathrm{C}$ and $\mathrm{a}_{\mathrm{i}}$ for wine acids were estimated according to the data presented in [8]. The activity coefficient of undissociated acids is assumed to be unity. All values of the dissociation constants necessary for calculations were taken from [9].

The concentrations of all charged and uncharged substances can be expressed by following equations:

$$
\begin{aligned}
& {\left[H^{+}\right]=c \sum_{m=1}^{n} \alpha_{m}=c \sum_{m=1}^{n} \alpha_{m}^{\prime} \alpha_{m-1}} \\
& {\left[H_{n-1} A^{-}\right]=c\left(\alpha_{1}-\alpha_{2}\right)=c \alpha_{1}\left(1-\alpha_{2}^{\prime}\right)} \\
& {\left[H_{n-2} A^{2-}\right]=c\left(\alpha_{2}-\alpha_{3}\right)=c \alpha_{1} \alpha_{2}^{\prime}\left(1-\alpha_{3}^{\prime}\right)} \\
& {\left[H_{n-m} A^{m-}\right]=c\left(\alpha_{m}-\alpha_{m+1}\right)=c \alpha_{1} \alpha_{2}^{\prime} \ldots \alpha_{m}^{\prime}\left(1-\alpha_{m+1}^{\prime}\right)} \\
& {\left[A^{n-}\right]=c \alpha_{n}=c \alpha_{1} \alpha_{2}^{\prime} \ldots \alpha_{n}^{\prime}} \\
& {\left[H_{n} A\right]=c\left(1-\alpha_{1}\right)}
\end{aligned}
$$

With the aid of Eqs. 4-8 we can determine the intervals of the acid concentration in which the various charged and uncharged substances dominate. This is possible according to the following equations expressed the conditions of equality of the various products of dissociation:

$$
\begin{aligned}
& {\left[H^{+}\right]=\left[H_{n} A\right]: \alpha_{1}=\frac{1-\sum_{m=2}^{n} \alpha_{m}}{2}} \\
& {\left[H_{n-1} A^{-}\right]=\left[H_{n} A\right]: \alpha_{1}=\frac{1+\alpha_{2}}{2} \text { or } \alpha_{1}=\frac{1}{2-\alpha_{2}^{\prime}}} \\
& {\left[H_{n-m} A^{m-}\right]=\left[H_{n} A\right]: \alpha_{1}=1-\alpha_{m}+\alpha_{m+1}}
\end{aligned}
$$

$$
\begin{aligned}
& \alpha_{1}=\frac{1}{1+\alpha_{2}^{\prime} \ldots \alpha_{m}^{\prime}\left(1-\alpha_{m+1}^{\prime}\right)} \\
& {\left[A^{n-}\right]=\left[H_{n} A\right]: \alpha_{1}=1-\alpha_{n} \text { or } \alpha_{1}=\frac{1}{1+\alpha_{2}^{\prime} \ldots \alpha_{m}^{\prime}}} \\
& {\left[H_{n-m} A^{m-}\right]=\left[H_{n-(m+1)} A^{(m+1)-}\right]: \alpha_{m}=2 \alpha_{m+1}-\alpha_{m+2}} \\
& \alpha_{m+1}^{\prime}=\frac{1}{2-\alpha_{m+2}}
\end{aligned}
$$

The $\left[\mathrm{H}^{+}\right]$value exceeds not only the concentration of any anion but also the sum of the concentrations of all anions which is equal to $\alpha_{1} c$.

\section{RESULTS AND DISCUSSION}

\subsection{Tartaric Acid}

Tartaric acid is, from a winemaking perspective, the most important wine acid due to the prominent role it plays in maintaining the chemical stability of the wine and its color and finally in influencing the taste of the finished wine. Usually the concentration of tartaric acid in wine is equal to $1-5 \mathrm{~g} \cdot \mathrm{dm}^{-3}$. The naturally occurring form of tartaric acid is L-tartaric acid.

Eq. 1 for both dissociation steps of weak dibasic organic acid may be written as follows:

$$
\begin{aligned}
& \mathrm{K}_{1}=\frac{c\left(\alpha_{1}^{2}-\alpha_{2}^{2}\right)}{1-\alpha_{1}} F_{1}=\frac{c \alpha_{1}^{2}\left[1-\left(\alpha_{2}^{\prime}\right)^{2}\right]}{1-\alpha_{1}} F_{1} \\
& \mathrm{~K}_{2}=\frac{c \alpha_{2}\left(\alpha_{1}+\alpha_{2}\right)}{\alpha_{1}-\alpha_{2}} F_{2}=\frac{c \alpha_{1} \alpha_{2}^{\prime}\left(1+\alpha_{2}^{\prime}\right)}{1-\alpha_{2}^{\prime}} F_{2}
\end{aligned}
$$

The degrees of dissociation $\alpha_{1}, \alpha_{2}$ and $\alpha_{2}^{\prime}$ may be evaluated successively by iterative solution of following quadratic equations:

$$
\begin{aligned}
& \alpha_{1}=\frac{1}{2}\left[-\frac{K_{1}}{c F_{1}}+\sqrt{\left(\frac{K_{1}}{c F_{1}}\right)^{2}+4\left(\alpha_{2}^{2}+\frac{K_{1}}{c F_{1}}\right)}\right] \\
& \alpha_{2}=\frac{1}{2}\left[-\left(\frac{K_{2}}{c F_{2}}+\alpha_{1}\right)+\sqrt{\left.\left(\frac{K_{2}}{c F_{2}}+\alpha_{1}\right)^{2}+\frac{4 K_{2} \alpha_{1}}{c F_{2}}\right]}\right. \\
& \alpha_{2}^{\prime}=\frac{1}{2}\left[-\left(1+\frac{K_{2}}{\alpha_{1} c F_{2}}\right)+\sqrt{\left(1+\frac{K_{2}}{\alpha_{1} c F_{2}}\right)^{2}+\frac{4 K_{2}}{\alpha_{1} c F_{2}}}\right]
\end{aligned}
$$


The $\left[\mathrm{H}^{+}\right],\left[\mathrm{HA}^{-}\right],\left[\mathrm{A}^{2-}\right]$ and $\left[\mathrm{H}_{2} \mathrm{~A}\right]$ values may be easily determined by Eqs. 4, 5, 8 and 9 .

The values of the dissociation constants for $\mathrm{L}$ tartaric acid are equal to: $\mathrm{K}_{1}=1.047 \times 10^{-3} ; \mathrm{K}_{2}=4.57 \times 10^{-5}$ $\left(25^{\circ} \mathrm{C}\right)$.

In Table 1 the values of the dissociation parameters of L-tartaric acid in its dilute $\left(0.0001-0.01 \mathrm{~mol} \cdot \mathrm{dm}^{-3}\right)$ solutions at $25^{\circ} \mathrm{C}$ calculated with the aid of above mentioned equations are presented.

The Eqs. 10, 11, 12 and 15 may be used for a determination the concentration intervals of prevailing of various dissociation products of tartaric acid. The concentration of monoanion exceeds the concentration of undissociated acid when $\mathrm{c} \leq 0.002 \mathrm{~mol} \cdot \mathrm{dm}^{-3}$ (the inequality $\alpha_{1}>\frac{1+\alpha_{2}}{2}$ is fulfilled). The $\left[\mathrm{A}^{2-}\right]$ value exceeds the $\left[\mathrm{H}_{2} \mathrm{~A}\right]$ value when $\mathrm{c} \leq 0.0002 \mathrm{~mol} \cdot \mathrm{dm}^{-3}$ (the inequality $\alpha_{1}>1-\alpha_{2}$ is fulfilled). Monoanion remains the predominant anion in all studied interval of concentrations of tartaric acid (the inequality $\alpha_{1}>2 \alpha_{2}$ or $\alpha_{2}^{\prime}<0.5$ are fulfilled). The $\left[\mathrm{H}^{+}\right]$value exceeds the $\left[\mathrm{H}_{2} \mathrm{~A}\right]$ value up to $\mathrm{c}=0.003 \mathrm{~mol} \cdot \mathrm{dm}^{-3}$ (in this interval the inequality $\alpha_{1}>\frac{1-\alpha_{2}}{2}$ is fulfilled).

Taking into account the comparative complexity of calculations by Eqs. 19-21, we suggest also the simple empirical equations for fast approximate calculation of the dissociation parameters of tartaric acid:

$\alpha_{1}=0.195 c^{-0.173}$

(for $\mathrm{c}=0.0001-0.001 \mathrm{~mol} \cdot \mathrm{dm}^{-3}$ ) $\alpha_{2}=1.8967 \times 10^{-4} c^{-0.793}$

(for $\mathrm{c}=0.0001-0.001 \mathrm{~mol} \cdot \mathrm{dm}^{-3}$ )

$\alpha_{2}^{\prime}=9.7275 \times 10^{-4} c^{-0.62}$

(for $\mathrm{c}=0.0001-0.002 \mathrm{~mol} \cdot \mathrm{dm}^{-3}$ )

$p H=0.962-0.738 \lg c$

(for $\mathrm{c}=0.0001-0.01 \mathrm{~mol} \cdot \mathrm{dm}^{-3}$ )

In the specified intervals of the tartaric acid concentration the values of the relative errors for Eqs. $22-25$ do not exceed $5 \%$ (the relative error is the ratio of the difference between the approximate value and corresponding accurate value, divided by the approximate value, and converted to percent).

\subsection{Malic Acid}

Malic acid, along with tartaric acid, is one of the major organic acids found in grapes and wine. It is found also in nearly every fruit and berry plant. In the grape vine, malic acid is involved in several processes which are essential for the health and sustainability of the vine. It is also responsible for the tart taste of green apples. This acid is one of the biologically fragile wine acids, and it is easily metabolized by several different types of wine bacteria. The concentration of malic acid in wine is equal to $1-6 \mathrm{~g} \cdot \mathrm{dm}^{-3}$.

The equations used for calculations in case of tartaric acid were also used for determination of the dissociation parameters of malic acid. The dissociation constants of malic acid have the following values: $\mathrm{K}_{1}=3.981 \times 10^{-4} ; \mathrm{K}_{2}=7.762 \times 10^{-6}\left(25^{\circ} \mathrm{C}\right)$. In Table 2 the values of the degrees of dissociation and $\mathrm{pH}$ for dilute solutions of malic acid at $25^{\circ} \mathrm{C}$ are presented.

Table 1: The Values of the Degrees of Dissociation and $\mathrm{pH}$ for Dilute Solutions of L-Tartaric Acid at 25C

\begin{tabular}{|c|c|c|c|c|}
\hline Concentration, $\mathbf{m o l} \cdot \mathbf{d m}^{-3}$ & $\boldsymbol{\alpha}_{1}$ & $\mathbf{\alpha}_{\mathbf{2}}$ & $\boldsymbol{\alpha}_{\mathbf{2}}$ & $\mathbf{p H}$ \\
\hline \hline 0.0001 & 0.928 & 0.274 & 0.295 & 3.926 \\
0.0002 & 0.866 & 0.166 & 0.192 & 3.693 \\
0.0003 & 0.819 & 0.122 & 0.149 & 3.558 \\
0.0004 & 0.780 & 0.0972 & 0.125 & 3.464 \\
0.0005 & 0.748 & 0.0809 & 0.108 & 3.393 \\
0.0006 & 0.720 & 0.0695 & 0.0965 & 3.335 \\
0.0007 & 0.695 & 0.0609 & 0.0876 & 3.288 \\
0.0008 & 0.674 & 0.0543 & 0.0806 & 3.246 \\
0.0009 & 0.654 & 0.0490 & 0.0749 & 3.211 \\
0.001 & 0.637 & 0.0447 & 0.0702 & 3.179 \\
0.002 & 0.520 & 0.0241 & 0.0463 & 2.978 \\
0.004 & 0.411 & 0.0140 & 0.0341 & 2.788 \\
0.006 & 0.353 & 0.00884 & 0.0250 & 2.684 \\
0.008 & 0.316 & 0.00678 & 0.0215 & 2.611 \\
0.01 & 0.289 & 0.00552 & 0.0191 & 2.554 \\
\hline
\end{tabular}


Table 2: The Values of the Degrees of Dissociation and $\mathrm{pH}$ for Dilute Solutions of Malic Acid at 25을

\begin{tabular}{|c|c|c|c|c|}
\hline Concentration, $\mathbf{m o l} \cdot \mathbf{d m}^{-3}$ & $\boldsymbol{\alpha}_{1}$ & $\boldsymbol{\alpha}_{2}$ & $\boldsymbol{\alpha}_{2}$ & $\mathbf{p H}$ \\
\hline \hline 0.0001 & 0.831 & 0.0688 & 0.0828 & 0.0505 \\
0.0002 & 0.736 & 0.0372 & 0.0382 & 3.051 \\
0.0003 & 0.672 & 0.0256 & 0.0314 & 3.687 \\
0.0004 & 0.624 & 0.0196 & 0.0272 & 3.597 \\
0.0005 & 0.586 & 0.0159 & 0.0242 & 3.530 \\
0.0006 & 0.555 & 0.0134 & 0.0219 & 3.476 \\
0.0007 & 0.529 & 0.0116 & 0.0202 & 3.431 \\
0.0008 & 0.507 & 0.0102 & 0.0187 & 3.393 \\
0.0009 & 0.488 & 0.00914 & 0.0176 & 3.360 \\
0.001 & 0.471 & 0.00827 & 0.0117 & 3.330 \\
0.002 & 0.366 & 0.00428 & 0.00794 & 3.143 \\
0.004 & 0.278 & 0.00221 & 0.00640 & 2.966 \\
0.006 & 0.235 & 0.00150 & 0.00550 & 2.866 \\
0.008 & 0.208 & 0.00114 & 0.00491 & 2.796 \\
\hline
\end{tabular}

With the aid of Eqs. 10, 11, 12 and 15 the concentration intervals of prevailing of the products of dissociation of malic acid were determined. The $\left[\mathrm{HA}^{-}\right]$ value exceeds the $\left[\mathrm{H}_{2} \mathrm{~A}\right]$ value up to $\mathrm{c}=0.0008 \mathrm{~mol}$. $\mathrm{dm}^{-3}$ (the inequality $\alpha_{1}>\frac{1+\alpha_{2}}{2}$ is fulfilled). The inequality $\alpha_{1}>1-\alpha_{2}$ is fulfilled in all intervals of the $\mathrm{c}$ values; this fact testifies that the $\left[\mathrm{A}^{2-}\right]$ value is always lower than $\left[\mathrm{H}_{2} \mathrm{~A}\right]$ value. The monoanion remains the predominant anion in all studied interval of the acid concentrations (the inequalities $\alpha_{1}>2 \alpha_{2}$ and $\alpha_{2}^{\prime}<0.5$ are fulfilled). The $\left[\mathrm{H}^{+}\right]$value exceeds the $\left[\mathrm{H}_{2} \mathrm{~A}\right]$ value up to $\mathrm{c}=0.0008 \mathrm{~mol} \cdot \mathrm{dm}^{-3}$ (in this interval the inequality $\alpha_{1}>\frac{1-\alpha_{2}}{2}$ is fulfilled).

The simple empirical equations for fast approximate calculation of the dissociation parameters of malic acid are presented below.

$\alpha_{1}=0.086124 c^{-0.25}$

(for $\mathrm{c}=0.0001-0.001 \mathrm{~mol} \cdot \mathrm{dm}^{-3}$ )

$\alpha_{2}=1.466 \times 10^{-5} c^{-0.92}$

(for $\mathrm{c}=0.0001-0.008 \mathrm{~mol} \cdot \mathrm{dm}^{-3}$ )

$\alpha_{2}^{\prime}=1.7022 \times 10^{-4} c^{-0.67}$

(for $\mathrm{c}=0.0001-0.002 \mathrm{~mol} \cdot \mathrm{dm}^{-3}$ )

$p H=1.188-0.713 \lg c$

(for $\mathrm{c}=0.0001-0.01 \mathrm{~mol} \cdot \mathrm{dm}^{-3}$ )

The values of the relative error for these equations in indicated concentration intervals do not exceed 5$6 \%$.

\subsection{Succinic Acid}

Succinic acid is most commonly found in wine but can also be present in trace amounts in ripened grapes. This acid is usually found in higher levels with red wine grapes. The concentration of succinic acid in wines is equal to $0.4-1 \mathrm{~g} \cdot \mathrm{dm}^{-3}$.

In Table 3 the values of the dissociation parameters of succinic acid for its dilute solutions at $25^{\circ} \mathrm{C}$ calculated with the aid of Eqs. 19-21 are presented. The dissociation constants for this acid have the following values: $\mathrm{K}_{1}=6.166 \times 10^{-5} ; \quad \mathrm{K}_{2}=2.291 \times 10^{-6}$ $\left(25^{\circ} \mathrm{C}\right)$.

The concentration of monoanion exceeds the concentration of undissociated succinic acid only when $\mathrm{c}=0.0001 \mathrm{~mol} \cdot \mathrm{dm}^{-3}$ (the inequality $\alpha_{1}>\frac{1+\alpha_{2}}{2}$ is fulfilled). The $\left[\mathrm{A}^{2-}\right]$ values are always lower than $\left[\mathrm{H}_{2} \mathrm{~A}\right]$ value (the inequality $\alpha_{1}<1-\alpha_{2}$ is fulfilled). The monoanion remains the predominant anion in all studied interval of the $\mathrm{c}$ values (the inequalities $\alpha_{1}>2 \alpha_{2}$ and $\alpha_{2}^{\prime}<0.5$ are fulfilled). The $\left[\mathrm{H}^{+}\right]$value exceeds the $\left[\mathrm{H}_{2} \mathrm{~A}\right]$ value only when $\mathrm{c}=0.0001 \mathrm{~mol}$. $\mathrm{dm}^{-3}$ (in this interval the inequality $\alpha_{1}>\frac{1-\alpha_{2}}{2}$ is fulfilled).

The simple empirical equations for fast approximate calculation of the dissociation parameters of succinic acid at $25^{\circ} \mathrm{C}$ are presented below.

$\alpha_{1}=0.0171 c^{-0.375}$ 
Table 3: The Values of the Degrees of Dissociation and pH for Dilute Solutions of Succinic Acid at 25C

\begin{tabular}{|c|c|c|c|c|}
\hline Concentration, $\mathbf{m o l} \cdot \mathbf{d m}^{-3}$ & $\boldsymbol{\alpha}_{1}$ & $\boldsymbol{\alpha}_{2}$ & $\boldsymbol{\alpha}_{2}^{\prime}$ & $\mathbf{p H}$ \\
\hline \hline 0.0001 & 0.539 & 0.0219 & 0.0406 & 0.0266 \\
0.0002 & 0.426 & 0.0113 & 0.0210 & 4.255 \\
0.0003 & 0.366 & 0.00769 & 0.0179 & 3.956 \\
0.0004 & 0.327 & 0.00583 & 0.0157 & 3.882 \\
0.0005 & 0.298 & 0.00470 & 0.0142 & 3.826 \\
0.0006 & 0.277 & 0.00394 & 0.0131 & 3.780 \\
0.0007 & 0.259 & 0.00339 & 0.0122 & 3.742 \\
0.0008 & 0.245 & 0.00298 & 0.0114 & 3.709 \\
0.0009 & 0.233 & 0.00266 & 0.0109 & 3.680 \\
0.001 & 0.219 & 0.00240 & 0.00748 & 3.661 \\
0.002 & 0.164 & 0.00123 & 0.00524 & 3.490 \\
0.004 & 0.119 & 0.000625 & 0.00426 & 3.329 \\
0.006 & 0.0989 & 0.000422 & 0.00369 & 3.236 \\
0.008 & 0.0864 & 0.000319 & 0.00331 & 3.171 \\
\hline
\end{tabular}

$\alpha_{2}=3.133 \times 10^{-6} c^{-0.962}$

(for $\mathrm{c}=0.0001-0.01 \mathrm{~mol} \cdot \mathrm{dm}^{-3}$ )

$\alpha_{2}^{\prime}=1.8323 \times 10^{-4} c^{-0.587}$

(for $\mathrm{c}=0.0001-0.002 \mathrm{~mol} \cdot \mathrm{dm}^{-3}$ )

$p H=1.89-0.589 \lg c$

(for $\mathrm{c}=0.0001-0.01 \mathrm{~mol} \cdot \mathrm{dm}^{-3}$ )

The values of the relative error for these equations in indicated concentration intervals do not exceed 5 $6 \%$.

\subsection{Citric Acid}

Only small amounts of citric acid are present in grapes and wines. Like malic acid, citric acid is easily converted into other materials by wine microorganisms. The concentration of this acid in wines is equal to 0.04 $0.5 \mathrm{~g} \cdot \mathrm{dm}^{-3}$.

Eq. 1 for three dissociation steps of tribasic citric acid may be written as follows:

$$
\begin{aligned}
& \mathrm{K}_{1}=\frac{\left(\alpha_{1}+\alpha_{2}+\alpha_{3}\right)\left(\alpha_{1}-\alpha_{2}\right) c}{1-\alpha_{1}} \\
& F_{1}=\frac{c \alpha_{1}^{2}\left(1+\alpha_{2}^{\prime}+\alpha_{2}^{\prime} \alpha_{3}^{\prime}\right)\left(1-\alpha_{2}^{\prime}\right)}{1-\alpha_{1}} F_{1} \\
& \mathrm{~K}_{2}=\frac{\left(\alpha_{1}+\alpha_{2}+\alpha_{3}\right)\left(\alpha_{2}-\alpha_{3}\right) c}{\alpha_{1}-\alpha_{2}} \\
& F_{2}=\frac{c \alpha_{1} \alpha_{2}^{\prime}\left(1+\alpha_{2}^{\prime}+\alpha_{2}^{\prime} \alpha_{3}^{\prime}\right)\left(1-\alpha_{3}^{\prime}\right)}{1-\alpha_{2}^{\prime}} F_{2}
\end{aligned}
$$

$$
\mathrm{K}_{3}=\frac{\left(\alpha_{1}+\alpha_{2}+\alpha_{3}\right) \alpha_{3} c}{\alpha_{2}-\alpha_{3}} F_{3}=\frac{c \alpha_{1} \alpha_{3}^{\prime}\left(1+\alpha_{2}^{\prime}+\alpha_{2}^{\prime} \alpha_{3}^{\prime}\right)}{1-\alpha_{3}^{\prime}} F_{3}
$$

The values of the usual degrees of dissociation, according to Eqs. 1-3, can be successively evaluated by an iterative solution of three quadratic equations:

$$
\begin{aligned}
& \alpha_{1}=\frac{1}{2}\left[-\left(\frac{K_{1}}{c F_{1}}+\alpha_{3}\right)+\sqrt{\left(\frac{K_{1}}{c F_{1}}+\alpha_{3}\right)^{2}+4\left(\alpha_{2}^{2}+\alpha_{2} \alpha_{3}+\frac{K_{1}}{c F_{1}}\right)}\right] \\
& \alpha_{2}=\frac{1}{2}\left[-\left(\frac{K_{2}}{c F_{2}}+\alpha_{1}\right)+\sqrt{\left(\frac{K_{2}}{c F_{2}}+\alpha_{1}\right)^{2}+4\left(\alpha_{3}^{2}+\alpha_{1} \alpha_{3}+\frac{K_{2} \alpha_{1}}{c F_{2}}\right)}\right] \\
& \alpha_{3}=\frac{1}{2}\left[-\left(\frac{K_{3}}{c F_{3}}+\alpha_{1}+\alpha_{2}\right)+\sqrt{\left(\frac{K_{3}}{c F_{3}}+\alpha_{1}+\alpha_{2}\right)^{2}+\frac{4 K_{3} \alpha_{2}}{c F_{3}}}\right]
\end{aligned}
$$

The values of "partial" degrees of dissociation may be calculated with the aid of following equations:

$$
\begin{aligned}
& \alpha_{2}^{\prime}=\alpha_{2} / \alpha_{1} \\
& \alpha_{3}^{\prime}=\alpha_{3} / \alpha_{2}
\end{aligned}
$$

because the expressions corresponding to Eqs. 37-39 have the comparatively complex forms.

The $\left[\mathrm{H}^{+}\right],\left[\mathrm{H}_{2} \mathrm{~A}^{-}\right],\left[\mathrm{HA}^{2-}\right],\left[\mathrm{A}^{3-}\right]$ and $\left[\mathrm{H}_{3} \mathrm{~A}\right]$ values may be easily calculated with the aid of Eqs. 4-6, 8 and 9.

The dissociation constants for three dissociation steps of citric acid have the following values: $\mathrm{K}_{1}=7.413 \times 10^{-4} ; \mathrm{K}_{2}=1.738 \times 10^{-5} ; \mathrm{K}_{3}=3.981 \times 10^{-7}\left(25^{\circ} \mathrm{C}\right)$. 
In Tables $\mathbf{4}$ and $\mathbf{5}$ the values of the dissociation parameters of citric acid in its dilute solutions at $25^{\circ} \mathrm{C}$ calculated with the aid of Eqs. 35-39 are presented.

The Eqs. 10-15 may be used for determination the concentration intervals of prevailing of various dissociation products of citric acid. Monoanion concentration exceeds the undissociated acid concentration when $\mathrm{c} \leq 0.001 \mathrm{~mol} \cdot \mathrm{dm}^{-3}$ (the inequality $\alpha_{1}>\frac{1+\alpha_{2}}{2}$ is fulfilled). The $\left[\mathrm{HA}^{2-}\right]$ value exceeds the $\left[\mathrm{H}_{3} \mathrm{~A}\right]$ value only at $\mathrm{c}=0.0001 \mathrm{~mol} \cdot \mathrm{dm}^{-3}$ (the inequality $\alpha_{1}>1-\alpha_{2}+\alpha_{3}$ is fulfilled). The trianion concentration in all studied concentration interval is lower then $\left[\mathrm{H}_{3} \mathrm{~A}\right]$ value (the inequality $\alpha_{1}>1-\alpha_{3}$ is fulfilled). Monoanion remains the predominant anion in all interval of the acid concentration (the inequality $\alpha_{1}>2 \alpha_{2}-\alpha_{3}$ is fulfilled). The $\left[\mathrm{H}^{+}\right]$value exceeds the $\left[\mathrm{H}_{3} \mathrm{~A}\right]$ value up to $\mathrm{c}=0.001$ $\mathrm{mol} \cdot \mathrm{dm}^{-3}$ (the inequality $\alpha_{1}>\frac{1-\alpha_{2}-\alpha_{3}}{2}$ is fulfilled).

The simple empirical equations for fast approximate calculation of the dissociation parameters of citric acid at $25^{\circ} \mathrm{C}$ are presented below.

$$
\begin{aligned}
& \alpha_{1}=0.15632 c^{-0.193} \\
& \left(\text { for } \mathrm{c}=0.0001-0.001 \mathrm{~mol} \cdot \mathrm{dm}^{-3}\right. \text { ) } \\
& \alpha_{2}=5.70164 \times 10^{-5} c^{-0.84} \\
& \text { (for } \mathrm{c}=0.0001-0.001 \mathrm{~mol} \cdot \mathrm{dm}^{-3} \text { ) }
\end{aligned}
$$

Table 4: The Values of the Usual Degrees of Dissociation for Dilute Solutions of Citric Acid at 25ㄷ

\begin{tabular}{|c|c|c|c|}
\hline Concentration, $\mathbf{m o l} \cdot \mathbf{d m}^{-3}$ & $\boldsymbol{\alpha}_{1}$ & $\boldsymbol{\alpha}_{2}$ & $\boldsymbol{\alpha}_{3}$ \\
\hline \hline 0.0001 & 0.897 & 0.126 & $5.24 \times 10^{-4}$ \\
0.0002 & 0.824 & 0.0769 & $1.87 \times 10^{-4}$ \\
0.0003 & 0.770 & 0.0542 & $9.73 \times 10^{-5}$ \\
0.0004 & 0.727 & 0.0420 & $6.13 \times 10^{-5}$ \\
0.0005 & 0.692 & 0.0344 & $4.29 \times 10^{-5}$ \\
0.0006 & 0.662 & 0.0292 & $3.21 \times 10^{-5}$ \\
0.0007 & 0.636 & 0.0253 & $2.52 \times 10^{-5}$ \\
0.0008 & 0.614 & 0.0224 & $2.03 \times 10^{-5}$ \\
0.0009 & 0.594 & 0.0201 & $1.69 \times 10^{-5}$ \\
0.001 & 0.576 & 0.0182 & $1.44 \times 10^{-5}$ \\
0.002 & 0.462 & 0.00956 & $4.94 \times 10^{-6}$ \\
0.004 & 0.359 & 0.00499 & $1.77 \times 10^{-6}$ \\
0.006 & 0.307 & 0.00341 & $1.00 \times 10^{-6}$ \\
0.008 & 0.273 & 0.00260 & $6.65 \times 10^{-7}$ \\
0.01 & 0.249 & 0.00211 & $4.86 \times 10^{-7}$ \\
\hline
\end{tabular}

Table 5: The Values of the "Partial" Degrees of Dissociation and pH for Dilute Solutions of Citric Acid at 25C

\begin{tabular}{|c|c|c|c|}
\hline Concentration, $\mathbf{m o l} \cdot \mathbf{d m}^{-3}$ & $\boldsymbol{\alpha}_{2}^{\prime}$ & $\boldsymbol{\alpha}_{3}^{\prime}$ & $\mathbf{p H}$ \\
\hline \hline 0.0001 & 0.140 & 0.00418 & 3.996 \\
0.0002 & 0.0934 & 0.00242 & 3.751 \\
0.0003 & 0.0704 & 0.00179 & 3.615 \\
0.0004 & 0.0578 & 0.00146 & 3.521 \\
0.0005 & 0.0497 & 0.00125 & 3.449 \\
0.0006 & 0.0440 & 0.00110 & 3.392 \\
0.0007 & 0.0398 & $9.94 \times 10^{-4}$ & 3.345 \\
0.0008 & 0.0365 & $9.04 \times 10^{-4}$ & 3.304 \\
0.0009 & 0.0338 & $8.43 \times 10^{-4}$ & 3.269 \\
0.001 & 0.0316 & $7.89 \times 10^{-4}$ & 3.237 \\
0.002 & 0.0207 & $5.17 \times 10^{-4}$ & 3.040 \\
0.004 & 0.0139 & $3.54 \times 10^{-4}$ & 2.854 \\
0.006 & 0.0111 & $2.94 \times 10^{-4}$ & 2.750 \\
0.008 & 0.00954 & $2.56 \times 10^{-4}$ & 2.678 \\
0.01 & 0.00849 & $2.30 \times 10^{-4}$ & 2.622 \\
\hline
\end{tabular}


$\alpha_{3}=2.799 \times 10^{-10} c^{-1.568}$

(for $\mathrm{c}=0.0001-0.002 \mathrm{~mol} \cdot \mathrm{dm}^{-3}$ )

$\alpha_{2}^{\prime}=2.9376 \times 10^{-4} c^{-0.677}$

(for $\mathrm{c}=0.0001-0.002 \mathrm{~mol} \cdot \mathrm{dm}^{-3}$ )

$\alpha_{3}^{\prime}=5.0298 \times 10^{-6} c^{-0.728}$

$\left(\right.$ for $\mathrm{c}=0.0001-0.001 \mathrm{~mol} \cdot \mathrm{dm}^{-3}$ )

$p H=1.000-0.744 \lg c$

(for $\mathrm{c}=0.0001-0.01 \mathrm{~mol} \cdot \mathrm{dm}^{-3}$ )

The relative error values for these equations do not exceed $5-6 \%$.

\section{REFERENCES}

Kvaratskhelia E, Kvaratskhelia R. J Solution Chem 6 2007;

[2] Kvaratskhelia E, Kvaratskhelia R. J Solution Chem 8 2008; 37: 1063-70.

[3] Kvaratskhelia E, Kvaratskhelia R. J Solution Chem 3 2009; 38: 345-49.

[4] Kvaratskhelia RK, Kvaratskhelia ER. Russ J Electrochem 2 2009; 45: 221-24.

[5] Kvaratskhelia E, Kvaratskhelia R. J Biomed Sci Eng 5 2010; 3: 484-87.

[6] Kvaratskhelia RK, Kvaratskhelia ER. Russ J Electrochem 8 2010; 46: 952-56.

[7] Kvaratskhelia E, Kvaratskhelia R. The electrolytic dissociation of benzenetricarboxylic acids. In: Burley KT, editor. Physical organic chemistry: new developments. New York: Nova Science Publishers 2010; pp. 171-182.

[8] Koryta J, Dvorak J, Kavan L. Principles of Electrochemistry. $2^{\text {nd }}$ ed. Chichester, New York, Brisbane, Toronto, Singapore: Wiley 1993.

[9] Lide DR. CRC Handbook of Chemistry and Physics. $81^{\text {st }}$ ed. Boca Raton, Ann Arbor, London, Tokyo: CRC Press 2001. 36: 787-92.

[1] 